\title{
High-Accuracy Carrier Phase Discriminator in One-Bit Quantized Software-Defined Receivers
}

\author{
Chieh-Fu Chang, Member, IEEE, and Ming-Seng Kao
}

\begin{abstract}
In this letter, a novel phase discriminator called digital phase discriminator (DPD) is proposed for 1-bit analog-to-digitalconversion (ADC) software-defined receivers. Using fewer computations, the DPD achieves higher accuracy than that of the typical arctangent phase discriminator (APD). The improvement is up to several orders when the sampling frequency keeps increasing since the accuracy of APD is bounded mainly due to quantization loss. Finally, the simulation results demonstrate the superiority of the proposed approach and the critical uniformity condition is verified.
\end{abstract}

Index Terms-Analog-to-digital-conversion (ADC), arctangent phase discriminator (APD), digital phase discriminator (DPD), phase discriminator, software-defined receiver.

\section{INTRODUCTION}

$\mathbf{T}$ HE idea of 1-bit analog-to-digital-conversion (ADC) have been investigated and widely implemented in GPS software-defined receiver and low-power satellite communications due to efficient bitwise processing and avoidance of automatic gain control [1]-[6]. In carrier synchronization, the noncoherent phase detection traditionally adopts arctangent as its phase discriminator which maximizes signal-to-noise-ratio (SNR) for analog signal case in additive-white-Gaussian-noise (AWGN) channel [7]. Since arctangent takes a significant computation at each tracking cycle for real-time implementation, a number of modifications were proposed in order to reduce the computation [8]. However, all of them are limited by specific constraints accordingly, and their accuracy degrades greatly because of 1-bit ADC, with the degradation larger than $2 \mathrm{~dB}$ in power for most applications [4], [9]. This is due to the high quantization loss associated with 1-bit quantization, which inevitably reduces the accuracy in phase estimation.

In this letter, a novel phase discriminator for software-defined receivers with 1-bit ADC was proposed. We directly utilize the polarity information provided by 1-bit ADC to estimate signal phase in order to avoid the significant loss in 1-bit quantization. This approach is developed based on digital perspective and hence called digital phase discriminator (DPD). It can be

Manuscript received November 12, 2007; revised February 3, 2008. The associate editor coordinating the review of this manuscript and approving it for publication was Dr. Xiang-Gen Xia.

C.-F. Chang is with the National Space Organization, Hsinchu 30078, Taiwan, R.O.C. (e-mail: jeffchang @nspo.org.tw).

M.-S. Kao is with the Department of Communication Engineering, National Chiao-Tung University, Hsinchu 30050, Taiwan, R.O.C. (e-mail: mskao@cc. nctu.edu.tw).

Color versions of one or more of the figures in this paper are available online at http://ieeexplore.iee.org.

Digital Object Identifier 10.1109/LSP.2008.922517

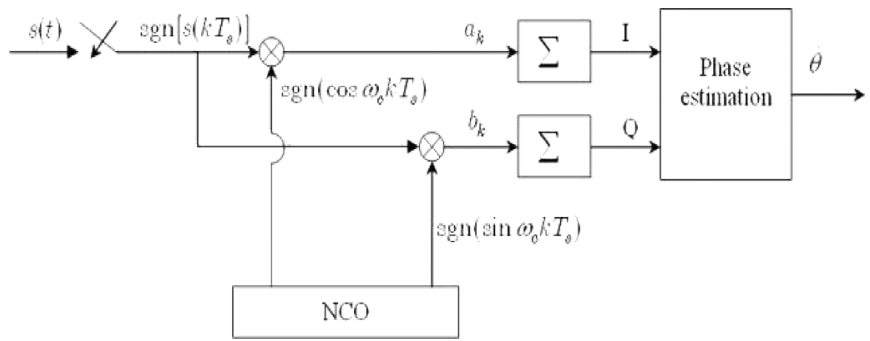

Fig. 1. Phase discriminator of a 1-bit software-defined receiver.

shown that the proposed DPD achieves much higher accuracy than that of traditional APD. Moreover, as linear function is used in phase estimation, the computation of our approach is also reduced comparing with the nonlinear arctangent method.

\section{Motivation}

Consider the phase discriminator in a typical 1-bit softwaredefined receiver as shown in Fig. 1. Let the received signal $s(t)$ be given by

$$
s(t)=A \cos \left(\omega_{c} t+\theta\right)+n(t)
$$

where $A$ is the signal amplitude, $\omega_{c}$ is the down-converted carrier frequency, $\theta$ is the unknown phase, and $n(t)$ is the added Gaussian noise. As phase detection is of interest, the output frequency of the numerical control oscillator $(\mathrm{NCO})$ is assumed to be equal to the incoming carrier frequency.

First, for the inphase branch, $a_{k}$ is given by

$$
\begin{aligned}
& a_{k}= \operatorname{sgn}\left[s\left(k T_{s}\right)\right] \cdot \operatorname{sgn}\left[\cos \omega_{c} k T_{s}\right] \\
&= \operatorname{sgn}\left[s\left(k T_{s}\right) \cdot \cos \omega_{c} k T_{s}\right] \\
&=\operatorname{sgn}\left[\frac{A}{2}\left[\cos \theta+\cos \left(2 \omega_{c} k T_{s}+\theta\right)\right]\right. \\
&\left.\quad+\cos \omega_{c} k T_{s} \cdot n\left(k T_{s}\right)\right]
\end{aligned}
$$

where $T_{s}$ is the sampling period and $\operatorname{sgn}[x]$ denotes the polarity function of $x$, i.e., $\operatorname{sgn}[\mathrm{x}]=1$ if $x \geq 0$ and $\operatorname{sgn}[\mathrm{x}]=-1$ if $x<0$. Similarly, for the quadrature branch, $b_{k}$ is given by

$$
b_{k}=\operatorname{sgn}\left[\frac{A}{2}\left[-\sin \theta+\sin \left(2 \omega_{c} k T_{s}+\theta\right)\right]+\sin \omega_{c} k T_{s} \cdot n\left(k T_{s}\right)\right] .
$$


Suppose $T$ is the observation time and $K=T / T_{s}$. Then the outputs of summations are given by

$$
\begin{gathered}
I=\sum_{k=0}^{K-1} \operatorname{sgn}\left[\frac{A}{2}\left[\cos \theta+\cos \left(2 \omega_{c} k T_{s}+\theta\right)\right]\right. \\
\left.+\cos \omega_{c} k T_{s} \cdot n\left(k T_{s}\right)\right] \\
Q=\sum_{k=0}^{K-1} \operatorname{sgn}\left[\frac{A}{2}\left[-\sin \theta+\sin \left(2 \omega_{c} k T_{s}+\theta\right)\right]\right. \\
\left.+\sin \omega_{c} k T_{s} \cdot n\left(k T_{s}\right)\right] .
\end{gathered}
$$

Finally, the phase is estimated using APD, given by

$$
\hat{\theta}=-A T N 2\left[\frac{Q}{I}\right]
$$

where $A T N 2(\cdot)$ denotes the arc-tangent-2 function. The summation process in (4) and (5) actually approximates integration in the analog domain. If all the signals in Fig. 1 are analog and the noise is neglected, the I-channel and Q-channel outputs of integrators become

$$
\begin{aligned}
I_{a} & =\int_{o}^{T} \frac{A}{2}\left[\cos \theta+\cos \left(2 \omega_{c} t+\theta\right)\right] d t \approx \frac{A T}{2} \cos \theta \\
Q_{a} & =\int_{o}^{T} \frac{A}{2}\left[-\sin \theta+\sin \left(2 \omega_{c} t+\theta\right)\right] d t \approx-\frac{A T}{2} \sin \theta
\end{aligned}
$$

where the high-frequency terms are neglected after the integration. Thus, the phase estimate using APD is given by

$$
\hat{\theta_{a}}=-A T N 2\left[\frac{Q_{a}}{I_{a}}\right]=\theta .
$$

Eqs. (4)-(6) approximate to (7)-(9) when $m$-bits quantization is adopted and $m \rightarrow \infty$. Unfortunately, when one-bit quantization is adopted, the estimated phase of (6) deviates from the real one due to significant quantization loss.

\section{PROPOSED APPROACH (DPD)}

Without loss of generality, we take $A / 2=1$ and neglect noise component in (2). We have

$$
a_{k}=\operatorname{sgn}\left[\cos \theta+\cos \phi_{k}\right]
$$

where

$$
\phi_{k}=2 \omega_{c} k T_{s}+\theta \bmod 2 \pi
$$

and $k=0,1,2 \ldots N-1$. Obviously, $a_{k}$ can be considered as the polarity of a discrete sinusoid plus a dc bias specified by $\cos \theta$. Assume $\phi_{k}$ 's are uniformly distributed over $[0,2 \pi]$, where

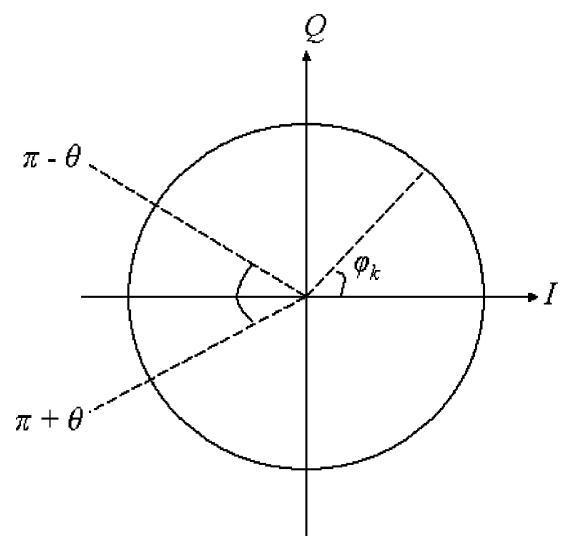

Fig. 2. Phase discriminator of a 1-bit quantized software-defined receiver.

$k=0,1,2 \ldots N-1$. Define the ratio of negative samples to the total number of samples within $T$ given by

$$
\eta_{i}=\frac{N_{-}}{N}
$$

where $N_{-}$is the number of samples with $a_{k}=-1$. From Fig. 2, when $0<\theta \leq \pi, a_{k}=-1$ only if $\phi_{k}$ is within the interval $[\pi-\theta, \pi+\theta]$. Since $\phi_{k}$ 's are uniformly distributed over $[0,2 \pi]$ if $N$ is sufficiently large, the parameter $\eta_{i}$ is approximated by

$$
\eta_{i} \approx \frac{2 \theta}{2 \pi}=\frac{\theta}{\pi}
$$

On the other hand, when $-\pi \leq \theta \leq 0, a_{k}=-1$ only if $\phi_{k}$ is within the interval $[\pi+\theta, \pi-\theta]$. In this case

$$
\eta_{i} \approx \frac{-2 \theta}{2 \pi}=\frac{-\theta}{\pi} .
$$

From (13) and (14), $\theta$ can be simply obtained from $\eta_{i}$ with polarity ambiguity. This ambiguity can be resolved when Q-channel signal is considered. Neglecting noise component in (3), we obtain

$$
b_{k}=\operatorname{sgn}\left[-\sin \theta+\sin \left(2 \omega_{c} k T_{s}+\theta\right)\right] \text {. }
$$

Define

$$
\eta_{q}=\frac{N_{-}^{\prime}}{N}
$$

where $N_{-}^{\prime}$ is the number of samples with $b_{k}=-1$. Following the same logic as deriving (13) and (14), we have

$$
\begin{array}{llll}
\eta_{q} \approx \frac{1}{2}+\frac{\theta}{\pi} & \text { if } & -\frac{\pi}{2} \leq \theta<\frac{\pi}{2} \\
\eta_{q} \approx \frac{3}{2}-\frac{\theta}{\pi} & \text { if } & \frac{\pi}{2} \leq \theta<\frac{3 \pi}{2} .
\end{array}
$$


From (17) and (18), we obtain

$$
\begin{array}{lll}
\eta_{q}>\frac{1}{2} \quad \text { if } & 0<\theta \leq \pi \\
\eta_{q} \leq \frac{1}{2} \quad \text { if } & -\pi<\theta \leq 0 .
\end{array}
$$

Clearly, the polarity of $\theta$ can be obtained directly from $\eta_{q}$. From (13), (14), (19), and (20), the phase can be derived by

$$
\theta=\operatorname{sgn}\left(\eta_{q}-\frac{1}{2}\right) \cdot \eta_{i} \pi
$$

Hence, we can simply use two negative sample counters (NSC) to count the number of negative samples of $a_{k}$ and $b_{k}$ and estimate the incoming carrier phase. From implementation viewpoint, the NSC result can be derived from summation outputs $I$ and $Q$ in Fig. 1 given by

$$
\eta_{i}=\frac{N-I}{2 N} \quad \eta_{q}=\frac{N-Q}{2 N}
$$

which enables the proposed approach to readily fit in the traditional structure.

\section{Simulation Results}

The phase estimate error of APD and proposed DPD via $\theta \in$ $[-\pi, \pi]$ are provided in Fig. 3 when $T=1 \mathrm{msec}$, carrier frequency $f_{c}=15.42 \mathrm{MHz}$, sampling frequency $f_{s}=512 \mathrm{KHz}$, and $4.096 \mathrm{MHz}$. From Fig. 3, in general, DPD phase error is much less than that of APD. Besides, both APD and DPD estimated errors exhibit periodic property and the transient variation via phase reduces when the sampling rate increases. In order to investigate the relationship between error performance and sampling frequency, we take the mean-squared error (MSE) over $\theta \in[-\pi, \pi]$ in both cases using different $f_{s}$ as shown in Fig. 4 and Table I. From Fig. 4, the phase error of APD is almost independent of $f_{s}$ when $f_{s} \geq 512 \mathrm{KHz}$. In contrast, the phase error of DPD constantly decreases as $f_{s}$ increases. It means that the accuracy of APD is bounded and DPD can attain several orders of improvement when the sampling rate keeps increasing.

\section{UNIFORMITY CONDITION}

In the proposed approach, the uniform assumption of $\phi_{k}$ 's over $[0,2 \pi]$ is a critical requirement to obtain the desired results. In the following, we are going to discuss this assumption. First, from (11) and for simplicity, let $\theta=0$. The carrier phase is given by

$$
\phi_{k}=2 \pi \frac{2 f_{c}}{f_{s}} k \bmod 2 \pi .
$$

In general, $2 f_{c} / f_{s}$ is a rational number written as

$$
\frac{2 f_{c}}{f_{s}}=n+\frac{q}{p}
$$
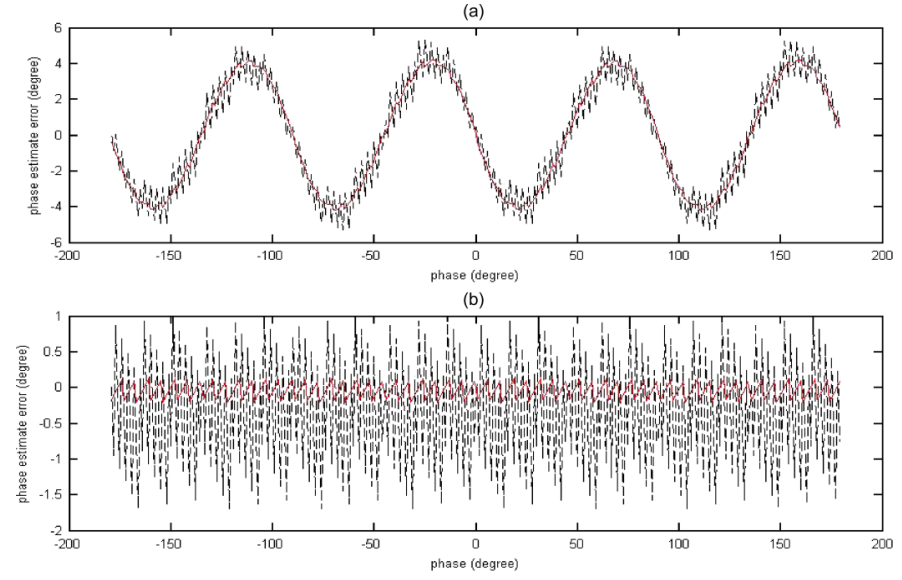

Fig. 3. Phase error of (a) typical APD and (b) proposed DPD when $T=$ $1 \mathrm{msec}, f_{c}=15.42 \mathrm{MHz}, f_{s}=512 \mathrm{KHz}$ (dashed line), and $4.096 \mathrm{MHz}$ (solid line).

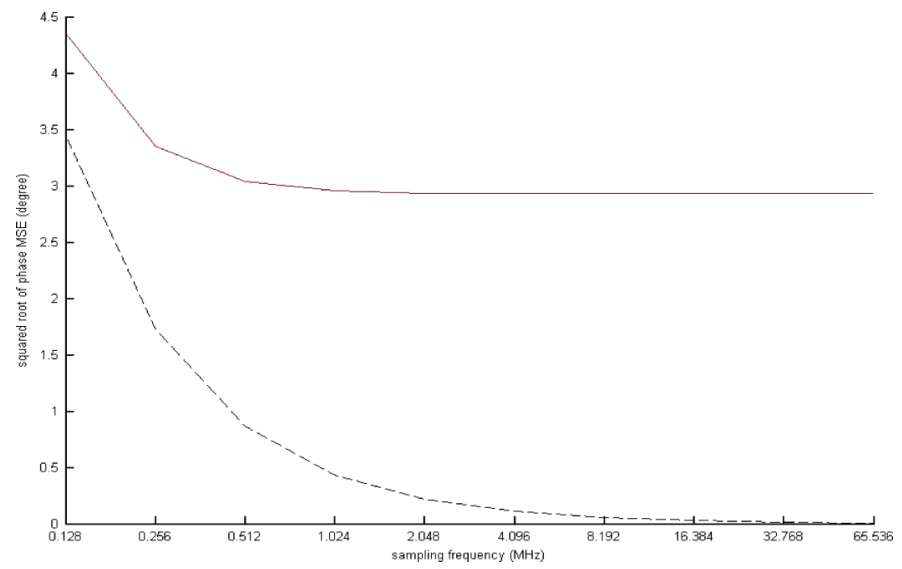

Fig. 4. Squared root of MSE using typical APD (solid line) and proposed DPD (dashed line) when $f_{s}=0.128 \mathrm{MHz}, 0.512 \mathrm{MHz} \ldots 65.536 \mathrm{MHz}$.

TABLE I

SQUARED ROOT OF APD MSE AND DPD MSE IN FIG. 4

\begin{tabular}{|c|c|c|c|c|c|c|c|c|c|c|}
\hline$f_{s}(\mathrm{MHZ})$ & 0.128 & 0.256 & 0.512 & 1.024 & 2.048 & 4.096 & 8.912 & 16.384 & 32.768 & 65.536 \\
\hline $\begin{array}{c}\text { Squared root } \\
\text { of APD MSE } \\
\text { (degree) }\end{array}$ & 4.352 & 3.347 & 3.034 & 2.951 & 2.934 & 2.929 & 2.928 & 2.928 & 2.927 & 2.927 \\
\hline $\begin{array}{c}\text { Squared root } \\
\text { of DPD MSE } \\
\text { (degree) }\end{array}$ & 3.451 & 1.725 & 0.8627 & 0.4314 & 0.2157 & 0.1078 & 0.0539 & 0.027 & 0.0135 & 0.0067 \\
\hline
\end{tabular}

where $n$ is the greatest integer less than or equal to $2 f_{c} / f_{s}$, while $p>q$ and they are relative prime integers. Obviously, $\phi_{k}$ is a periodic function with the period $p$. Hence, we only need to consider $\phi_{k}$, where $k=0,1,2 \ldots p-1$. Next, we have the following lemma.

Lemma 1: For any $i, j<p$ and $i \neq j,\left|\phi_{i}-\phi_{j}\right| \geq 2 \pi / p$.

Proof: Without loss of generality, assume $i>j$. Then

$$
\left|\phi_{i}-\phi_{j}\right|=\frac{2 \pi}{p} \cdot q \cdot(i-j) \bmod 2 \pi
$$


Let $h$ be the greatest common divider of $p$ and $i-j$, and

$$
p=u \cdot h \quad i-j=v \cdot h .
$$

Hence

$$
\left|\phi_{i}-\phi_{j}\right|=\frac{2 \pi}{u} \cdot q \cdot v \bmod 2 \pi .
$$

Since $(u, v)$ are relative prime integers and so are $(u, q)$, it follows

$$
\left|\phi_{i}-\phi_{j}\right| \geq \frac{2 \pi}{u} \geq \frac{2 \pi}{p} .
$$

The only distribution of $\phi_{k}$, where $k=0,1,2 \ldots p-1$, satis-

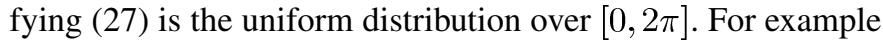
in our simulation case, $f_{c}=15.42 \mathrm{MHz}$ and $f_{s}=4.096 \mathrm{MHz}$. This corresponds to $n=7, q=271$, and $p=512$ in (24). As $T=1 \mathrm{msec}$ and the value of observations within $T$ is 4096 , which is the multiple of $p$. The uniform condition in Lemma 1 is satisfied and $p$ is sufficiently large to obtain accurate results.

\section{DISCUSSIONS AND CONCLUSION}

In noiseless (the noise effect can be neglected compared with quantization loss) environments, the proposed DPD has been shown to achieve higher accuracy than traditional APD in 1-bit software-defined receivers up to several orders since the accuracy of APD is bounded as shown in Fig. 4. Hence, the proposed phase discriminator may be applied in high-accuracy ranging analysis or some high-precision instrumentations. Besides, the DPD can be readily derived via the traditional structure from (21) and (22), and the computation is reduced compared with nonlinear arctangent discriminator. This feature makes the proposed phase discriminator very attractive in real-time processing receivers.

For noisy environment, we must consider noise effect and modify the DPD in (21), which is illustrated in Fig. 5 using Monte Carlo simulation for 100 trials. From this example, the proposed DPD is more sensitive to the noise than traditional APD, and the performance may be worse than APD in low SNR cases. In order to broaden the applications of DPD, a modified DPD having noise robust characteristic is under investigation.

Next, the DPD actually utilizes digital sampling output of 1-bit ADC to extract phase information. The "flavor" is similar to recent frequency delta-sigma frequency discriminator which converts instantaneous frequency deviation to digital samples [10]-[12]. The incorporation of each other may benefit mutually and enlarge the associated applications.

Finally, this letter also reveals the periodic characteristics of error performance in 1-bit software-defined receiver as shown in (a)

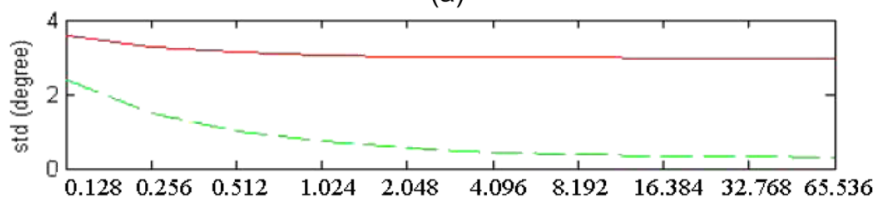

(b)

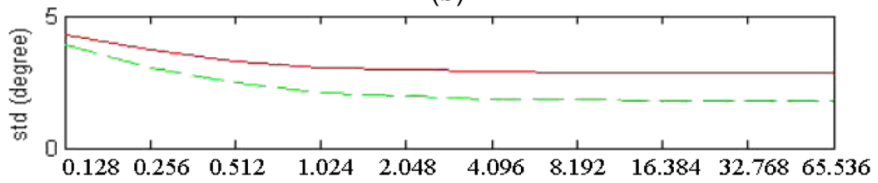

(c)

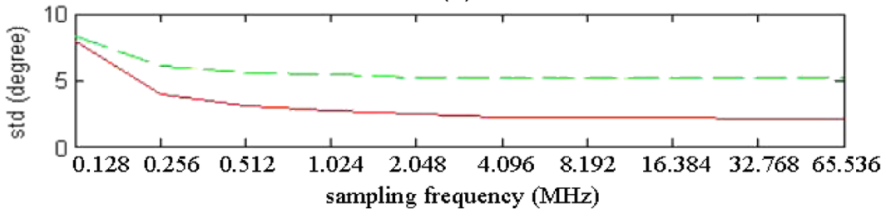

Fig. 5. Squared root of phase estimate MSE using typical APD (solid line) and proposed DPD (dashed line) in noisy environments when SNR = (a) $20 \mathrm{~dB}$, (b) $10 \mathrm{~dB}$ and (c) $5 \mathrm{~dB}$ using Monte Carlo simulation for 100 trials.

Fig. 3. These characteristics are consistent with our intuition as we consider the incoming sinusoidal wave as a periodic square wave in digital perspective. More interesting characteristics are worth exploring and would be our next step.

\section{REFERENCES}

[1] T. C. Marek, "Performance of ASCAMP DPSK downlink processor," in Proc. MILCOM '94, 1994, pp. 519-523.

[2] P. H. Wu, "The optimal BPSK demodulator with a 1-bit A/D frontend," in Proc. MILCOM '98, 1998, pp. 730-735.

[3] A. Brown and B. Wolt, "Digital L-band receiver architecture with direct RF sampling," in Proc. IEEE Position Location and Navigation Symp., Apr. 1994, pp. 209-216.

[4] B. Parkinson and J. Spilker, "Global positioning system: Theory and applications," AIAA, vol. I, 1996.

[5] B. Ledvina and M. Psiaki et al., "A real-time GPS civilian L1/L2 software receiver," in Proc. ION GNSS 2004, Sep. 2004, pp. 986-1005.

[6] C.-F. Chang, R.-M. Yang, M.-S. Kao, and J.-C. Juang, "A novel scheme and modified structures for improved software GPS Receiver," in Proc. ION GNSS 2007, Sep. 2007.

[7] J. G. Proakis, Digital Communications, 3rd ed. New York: McGrawHill, 1995.

[8] E. D. Kaplan and C. J. Hegarty, Understanding GPS Principles and Applications, 2nd ed. Norwood, MA: Artech House, 2006.

[9] H. Chang, "Presampling filtering, sampling and quantization effects on the digital matched filter performance," in Proc. Int. Telemetering Conf., San Diego, CA, Sep. 1982, pp. 889-915.

[10] J. C. Candy and G. C. Temes, Oversampling Delta-Sigma Data Converters. New York: IEEE Press, 1992.

[11] M. Hovin, A. Olsen, T. Lande, and C. Toumazou, "Delta-Sigma converters using frequency-modulated intermediate values," IEEE J. SolidState Circuits, vol. 32, no. 1, pp. 13-22, Jan. 1997.

[12] J. Kwon and B. Bakkaloglu, "Impact of sampling clock phase noise on $\sigma \delta$ frequency discriminators," IEEE Trans. Circuits Syst., vol. 54, no. 11, pp. 949-953, Nov. 2007. 